\title{
Analytical Solutions of Ionic Diffusion and Heat Conduction in Multilayered Porous Media
}

\author{
Yu Bai, ${ }^{1}$ Ali Harajli, ${ }^{2}$ and Yunping $\mathrm{Xi}^{3}$ \\ ${ }^{1}$ Beijing University of Civil Engineering and Architecture, Beijing 100044, China \\ ${ }^{2}$ Colorado Department of Transportation, Denver, CO, USA \\ ${ }^{3}$ University of Colorado Boulder, Boulder, CO, USA
}

Correspondence should be addressed to Yunping Xi; yunping.xi@colorado.edu

Received 25 June 2015; Revised 4 November 2015; Accepted 4 November 2015

Academic Editor: Goangseup Zi

Copyright (c) $2015 \mathrm{Yu}$ Bai et al. This is an open access article distributed under the Creative Commons Attribution License, which permits unrestricted use, distribution, and reproduction in any medium, provided the original work is properly cited.

\begin{abstract}
Ionic diffusion and heat conduction in a multiple layered porous medium have many important engineering applications. One of the examples is the chloride ions from deicers penetrating into concrete structures such as bridge decks. Different overlays can be placed on top of concrete surface to slowdown the chloride penetration. In this paper, the chloride ion diffusion equations were established for concrete structures with multiple layers of protective system. By using Laplace transformation, an analytical solution was developed first for chloride concentration profiles in two-layered system and then extended to multiple layered systems with nonconstant boundary conditions, including the constant boundary and linear boundary conditions. Because ionic diffusion in saturated media and heat conduction are governed by the same form of partial differential equations with different materials parameters, the analytical solution was further extended to handle heat conduction in a multiple layered system under nonconstant boundary conditions. The numerical results were compared with available test data. The basic trends of the analytical solution and the test data agreed quite well.
\end{abstract}

\section{Introduction}

Ionic diffusion and heat conduction in a multiple layered porous medium have many engineering applications. One of the examples is the chloride ion diffusion into concrete structures such as bridge decks. This topic will be one of the engineering examples used in this study. The basic formulation and solution of the partial differential equations will be based on the chloride ion diffusion in concrete structures. The chloride ions come from deicers used in the winter maintenance of concrete pavements and bridges. The chloride ions penetrate into concrete and reach the embedded steel bars (called rebars). Once the concentration of the chloride ions at the rebar level reaches a critical level, the rebar corrosion process will start. Rebar corrosion can cause the reduction of service life of reinforced concrete structures by two detrimental effects: the reduction of cross section area of rebars and the spalling of concrete cover due to the formation of rust (the density of rust is smaller than that of steel). Corrosion damage of reinforced concrete structures is a widespread phenomenon all over the world. Many protective measures have been developed and applied. For concrete bridges, different types of overlays can be installed on the top surface of reinforced concrete bridge decks to slow down the penetration of chloride ions, such as thin-bonded polymer overlays and asphaltic waterproof membranes. The diffusion resistances of the overlay systems and the concrete of bridge decks are considerably different, because they are made of different materials. Therefore, there is a pressing need to predict the chloride diffusion in concrete structures with multiple layers of different protective systems, which is the main topic of this paper. Much research has been conducted on the topic of ionic diffusion in porous media [1-5]. The efforts were spent on different topics such as modelling of diffusion parameters and effect of ionic and moisture interactions during the diffusion process. There has been no analytical solution available for ionic diffusion in multiple layered systems. 


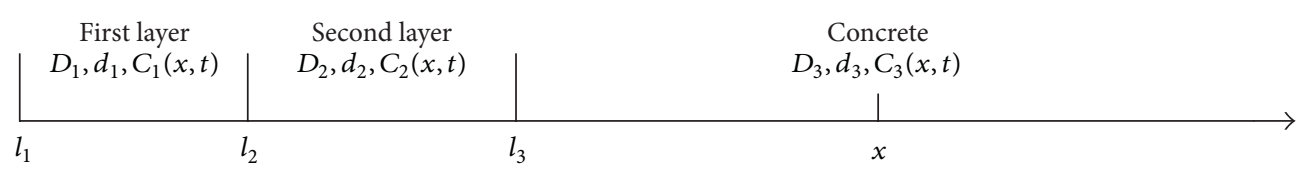

FIGURE 1: Concrete with two layers of protection.

Heat conduction in concrete structures is also important in practice and its mathematical description is very much similar to the chloride diffusion in concrete. There are many available solutions for heat conduction in different systems with different boundary conditions [6]. But, there has been no analytical solution available for heat conduction in multiple layered systems. Since the governing equations for the heat conduction and ionic diffusion have the same format but with different material parameters, the solutions for the ionic diffusion problem can also be used for the heat conduction problem.

In this paper, we will first present the diffusion model with two protective layers on top of concrete in Section 2. In Section 3, we will use Laplace transform to obtain the analytical solution of the diffusion equation under constant boundary conditions. In Section 4, we will change the boundary conditions to nonconstant conditions and obtain the corresponding analytical solutions. We will then generalize our results to the case with $(n-1)$ layers of protective system in Section 5. In Section 6, we will expand our results to the heat conduction equation. A numerical solution of heat condition will be given and available test data will be used to verify the present analytical solutions. Finally, conclusions will be given in Section 7 .

\section{Two Protective Layers under Constant Boundary Conditions}

The diffusion of chloride ions in concrete can be represented by Fick's first law. The flux of chloride ions through a concrete structure is proportional to the gradient of free chloride concentration in the pore solution in concrete:

$$
J=-D_{\mathrm{cl}} \operatorname{grad}\left(C_{f}\right),
$$

where $J$ is the flux of free chloride ions, $D_{\mathrm{cl}}$ is the chloride diffusivity, and $C_{f}$ is the free chloride concentration in the pore solution. Once chloride ions get into concrete, some of them attached to the surface of pore wall and the others are free to move forward. Apparently, the flux of chloride ions is related only to the free chloride ion gradient in (1). The total chloride ions are the sum of the attached ions (called bounded ions) and the free ions. Combining (1) with the mass conservation law $([1,2])$, we can obtain Fick's second law:

$$
\frac{d C_{t}}{d t}=-\operatorname{div}(J)
$$

in which $t$ is time in days and $C_{t}$ is the total chloride concentration. By substituting (2) into (1) and decoupling the free chloride concentration from the total chloride concentration, the governing equation of the diffusion of chlorides into saturated concrete can be written as $([3-5])$

$$
\frac{\partial C_{t}}{\partial t}=\frac{\partial C_{t}}{\partial C_{f}} \frac{\partial C_{f}}{\partial t}=\operatorname{div}\left[D_{\mathrm{cl}} \operatorname{grad}\left(C_{f}\right)\right]
$$

Governing partial differential equation (3) includes two material parameters: the chloride binding capacity $\left(\partial C_{f} / \partial C_{t}\right)$ and the chloride diffusivity $\left(D_{\mathrm{cl}}\right)$. Both parameters depend on the properties of concrete and the environmental conditions.

In this paper we assumed that the two material parameters $D_{\mathrm{cl}}$ and $\left(\partial C_{f} / \partial C_{t}\right)$ are two constants, and thus a combined parameter can be defined $-d_{\mathrm{cl}}=D_{\mathrm{cl}}\left(\partial C_{f} / \partial C_{t}\right)-$ which is called the chloride diffusion coefficient. The threedimensional diffusion equation, (3), can be written in a onedimensional form:

$$
\frac{\partial C_{f}}{\partial t}=d_{\mathrm{cl}} \frac{\partial^{2} C_{f}}{\partial x^{2}}
$$

in which $d_{\mathrm{cl}}$ is the chloride diffusion coefficient. As mentioned earlier, we will use chloride ion penetration into concrete bridge desk as an example. There are two layers of protection on the bridge decks as shown in Figure 1. Subscript 1 is for the top layer, 2 for the second layer, and 3 for the substrate (i.e., the concrete deck). We denote that the chloride diffusion coefficients of the first layer, the second layer, and concrete are $d_{1}, d_{2}$, and $d_{3}$, respectively, which are all constants, and the corresponding concentrations of chloride ions are $C_{1}(x, t), C_{2}(x, t)$, and $C_{3}(x, t)$, respectively. Based on Fick's first and second laws, the concentrations of chlorides ions in the first layer, the second layer, and the concrete satisfy the following differential equations:

$$
\begin{array}{ll}
\frac{\partial C_{1}}{\partial t}=d_{1} \frac{\partial^{2} C_{1}}{\partial x^{2}}, & l_{1} \leq x \leq l_{2}, \\
\frac{\partial C_{2}}{\partial t}=d_{2} \frac{\partial^{2} C_{2}}{\partial x^{2}}, & l_{2} \leq x \leq l_{3}, \\
\frac{\partial C_{3}}{\partial t}=d_{3} \frac{\partial^{2} C_{3}}{\partial x^{2}}, & x \geq l_{3} .
\end{array}
$$

There are two interfaces in the system: one is between two layers of overlays and the other one is between the second overlay and the concrete. At the interfaces, not only the concentrations of chlorides ions, but also the fluxes of 
chloride ions should be equal (the continuity conditions). That is,

$$
\begin{aligned}
C_{1}\left(l_{2}, t\right) & =C_{2}\left(l_{2}, t\right), \\
\left.D_{1} \frac{\partial C_{1}}{\partial x}\right|_{x=l_{2}} & =\left.D_{2} \frac{\partial C_{2}}{\partial x}\right|_{x=l_{2}}, \\
C_{2}\left(l_{3}, t\right) & =C_{3}\left(l_{3}, t\right), \\
\left.D_{2} \frac{\partial C_{2}}{\partial x}\right|_{x=l_{3}} & =\left.D_{3} \frac{\partial C_{3}}{\partial x}\right|_{x=l_{3}} .
\end{aligned}
$$

Assume the initial conditions are

$$
C_{1}(x, 0)=C_{2}(x, 0)=C_{3}(x, 0)=C_{0} .
$$

The boundary conditions are

$$
\begin{aligned}
& C_{1}\left(l_{1}, t\right)=C_{s}, \\
& C_{1}(\infty, t)<\infty, \\
& C_{2}(\infty, t)<\infty, \\
& C_{3}(\infty, t)=C_{0} .
\end{aligned}
$$

$C_{0}$ and $C_{s}$ are initial and boundary concentrations, respectively. The diffusion model with two layers of protection is set up by (5) to (14).

\section{Analytical Solution for \\ the Two-Protective-Layered System}

In this section we use Laplace transform to obtain the analytical solution of (5)-(14).

3.1. Laplace Transform for the First Layer. Denote the Laplace transform of $C_{1}(x, t)$ by $\bar{C}_{1}(x, p)$; that is,

$$
\bar{C}_{1}(x, p)=\mathscr{L}\left(C_{1}(x, t)\right)=\int_{0}^{\infty} C_{1}(x, t) e^{-p t} d t .
$$

Then we use Laplace transform for the equations of the first layer, which are (5), (12), (13), and (14), and we have

$$
\begin{aligned}
\frac{d^{2}}{d x^{2}} \bar{C}_{1}(x, p) & =\frac{p}{d_{1}} \bar{C}_{1}(x, p)-\frac{C_{0}}{d_{1}} \\
\bar{C}_{1}\left(l_{1}, p\right) & =\frac{C_{s}}{p} \triangleq \bar{C}_{s} .
\end{aligned}
$$

Suppose $J_{0}$ is the flux of chloride ions at $l_{1}$. By using (1), we have

$$
J_{0}=-\left.D_{1} \frac{d C_{1}}{d x}\right|_{x=l_{1}}
$$

By the Laplace transform of (18), we have

$$
\frac{d}{d x} \bar{C}_{1}\left(l_{1}, p\right)=-\frac{\bar{J}_{0}}{D_{1}},
$$

where $\bar{J}_{0}$ is the Laplace transform of $J_{0}$, which will be determined later. Denote $q_{1}=\sqrt{p / d_{1}}$. The solution of (16) and (17) is

$$
\bar{C}_{1}(x, p)=s_{1} e^{-q_{1} x}+s_{2} e^{q_{1} x}+\frac{C_{0}}{p}
$$

$$
s_{1}, s_{2} \text { to be determined. }
$$

Substitute (16) and (17) into (20); we have

$$
\begin{aligned}
\bar{C}_{s}-\frac{C_{0}}{p} & =s_{1} e^{-q_{1} l_{1}}+s_{2} e^{q_{1} l_{1}}, \\
-\frac{\bar{J}_{0}}{D_{1}} & =-q_{1} s_{1} e^{-q_{1} l_{1}}+q_{1} s_{2} e^{q_{1} l_{1}} .
\end{aligned}
$$

And then

$$
\begin{aligned}
& s_{1}=\frac{\left(\bar{C}_{s}-C_{0} / p\right)+\bar{J}_{0} / D_{1} q_{1}}{2} e^{-q_{1}\left(x-l_{1}\right)}, \\
& s_{2}=\frac{\left(\bar{C}_{s}-C_{0} / p\right)-\bar{J}_{0} / D_{1} q_{1}}{2} e^{q_{1}\left(x-l_{1}\right)} .
\end{aligned}
$$

Consequently,

$$
\begin{aligned}
\bar{C}_{1}(x, p)-\frac{C_{0}}{p}= & \left(\bar{C}_{s}-\frac{C_{0}}{p}\right) \cosh q_{1}\left(x-l_{1}\right) \\
& -\frac{\bar{J}_{0}}{D_{1} q_{1}} \sinh q_{1}\left(x-l_{1}\right),
\end{aligned}
$$

and, by using (1),

$$
\begin{aligned}
\bar{J}_{1}(x, p)= & -D_{1} \frac{d \bar{C}_{1}}{d x} \\
= & -D_{1}\left(\bar{C}_{s}-\frac{C_{0}}{p}\right) q_{1} \sinh q_{1}\left(x-l_{1}\right) \\
& +\bar{J}_{0} \cosh q_{1}\left(x-l_{1}\right)
\end{aligned}
$$

where $\bar{J}_{1}$ is the Laplace transform of $J_{1}$, the flux of chloride ions in the first layer.

Using the matrix format [6], we have

$$
\left(\begin{array}{c}
\bar{C}_{1}(x, p)-\frac{C_{0}}{p} \\
\bar{J}_{1}(x, p)
\end{array}\right)=\left(\begin{array}{cc}
\cosh q_{1}\left(x-l_{1}\right) & -\frac{1}{D_{1} q_{1}} \sinh q_{1}\left(x-l_{1}\right) \\
-D_{1} q_{1} \sinh q_{1}\left(x-l_{1}\right) & \cosh q_{1}\left(x-l_{1}\right)
\end{array}\right)\left(\begin{array}{c}
\bar{C}_{s}(x, p)-\frac{C_{0}}{p} \\
\bar{J}_{0}(x, p)
\end{array}\right) .
$$


3.2. Laplace Transform for the Second Layer. Denote the Laplace transform of $C_{2}(x, t)$ by $\bar{C}_{2}(x, p)$; we can use Laplace transform for the equations of the second layer, which are (6), (8), (9), (12), and (14), and we have

$$
\begin{gathered}
\frac{d^{2}}{d x^{2}} \bar{C}_{2}(x, p)=\frac{p}{d_{2}} \bar{C}_{2}(x, p)-\frac{C_{0}}{d_{2}}, \quad \begin{array}{l}
\text { ions in the second layer. Denote } q_{2}=\sqrt{p / d_{2}} \cdot \text { With }(25),(27), \\
\text { and }(28), \text { we have }
\end{array} \\
\left(\begin{array}{c}
\bar{C}_{2}\left(l_{2}, p\right)-\frac{C_{0}}{p} \\
\bar{J}_{2}\left(l_{2}, p\right)
\end{array}\right)=\left(\begin{array}{c}
\bar{C}_{1}\left(l_{2}, p\right)-\frac{C_{0}}{p} \\
\bar{J}_{1}\left(l_{2}, p\right)
\end{array}\right)=\left(\begin{array}{cc}
\cosh q_{1}\left(l_{2}-l_{1}\right) & -\frac{1}{D_{1} q_{1}} \sinh q_{1}\left(l_{2}-l_{1}\right) \\
-D_{1} q_{1} \sinh q_{1}\left(l_{2}-l_{1}\right) & \cosh q_{1}\left(l_{2}-l_{1}\right)
\end{array}\right)\left(\begin{array}{c}
\bar{C}_{s}-\frac{C_{0}}{p} \\
\bar{J}_{0}
\end{array}\right) \cdot\left(\begin{array}{c}
(29)
\end{array}\right.
\end{gathered}
$$

$$
\begin{gathered}
\bar{C}_{1}\left(l_{2}, p\right)=\bar{C}_{2}\left(l_{2}, p\right), \\
\bar{J}_{1}\left(l_{2}, p\right)=\bar{J}_{2}\left(l_{2}, p\right),
\end{gathered}
$$

where $\bar{J}_{2}$ is the Laplace transform of $J_{2}$, the flux of chloride

Using the similar method in Section 3.1, we have

$$
\left(\begin{array}{c}
\bar{C}_{2}(x, p)-\frac{C_{0}}{p} \\
\bar{J}_{2}(x, p)
\end{array}\right)=\left(\begin{array}{cc}
\cosh q_{2}\left(x-l_{2}\right) & -\frac{1}{D_{2} q_{2}} \sinh q_{2}\left(x-l_{2}\right) \\
-D_{2} q_{2} \sinh q_{2}\left(x-l_{2}\right) & \cosh q_{2}\left(x-l_{2}\right)
\end{array}\right)\left(\begin{array}{c}
\bar{C}_{1}\left(l_{2}, p\right)-\frac{C_{0}}{p} \\
\bar{J}_{1}\left(l_{2}, p\right)
\end{array}\right)
$$

3.3. Laplace Transform of the Last Layer (the Concrete Deck). Denote the Laplace transform of $C_{3}(x, t)$ by $\bar{C}_{3}(x, p)$; we can use Laplace transform for the equations of the last layer, the concrete, which are (7), (10), (11), (12), and (14); we have

$$
\begin{aligned}
& \frac{d^{2}}{d x^{2}} \bar{C}_{3}(x, p)=\frac{p}{d_{3}} \bar{C}_{2}(x, p)-\frac{C_{0}}{d_{3}} \\
& \left(\begin{array}{c}
\bar{C}_{3}\left(l_{3}, p\right)-\frac{C_{0}}{p} \\
\bar{J}_{3}\left(l_{3}, p\right)
\end{array}\right)=\left(\begin{array}{c}
\bar{C}_{2}\left(l_{3}, p\right)-\frac{C_{0}}{p} \\
\bar{J}_{2}\left(l_{3}, p\right)
\end{array}\right)=\left(\begin{array}{cc}
\cosh q_{2}\left(l_{3}-l_{2}\right) & -\frac{1}{D_{2} q_{2}} \sinh q_{2}\left(l_{3}-l_{2}\right) \\
-D_{2} q_{2} \sinh q_{2}\left(l_{3}-l_{2}\right) & \cosh q_{2}\left(l_{3}-l_{2}\right)
\end{array}\right)\left(\begin{array}{c}
\bar{C}_{1}\left(l_{2}, p\right)-\frac{C_{0}}{p} \\
\bar{J}_{1}\left(l_{2}, p\right)
\end{array}\right) .
\end{aligned}
$$

$$
\begin{aligned}
\bar{C}_{3}(\infty, p) & =\frac{C_{0}}{p}, \\
\bar{C}_{2}\left(l_{3}, p\right) & =\bar{C}_{3}\left(l_{3}, p\right), \\
\bar{J}_{2}\left(l_{3}, p\right) & =\bar{J}_{3}\left(l_{3}, p\right),
\end{aligned}
$$

Using the similar method in Section 3.1, we have

$$
\left(\begin{array}{c}
\bar{C}_{3}(x, p)-\frac{C_{0}}{p} \\
\bar{J}_{3}(x, p)
\end{array}\right)=\left(\begin{array}{cc}
\cosh q_{3}\left(x-l_{3}\right) & -\frac{1}{D_{3} q_{3}} \sinh q_{3}\left(x-l_{3}\right) \\
-D_{3} q_{3} \sinh q_{3}\left(x-l_{3}\right) & \cosh q_{3}\left(x-l_{3}\right)
\end{array}\right)\left(\begin{array}{c}
\bar{C}_{3}\left(l_{3}, p\right)-\frac{C_{0}}{p} \\
\bar{J}_{3}\left(l_{3}, p\right)
\end{array}\right) .
$$

3.4. Solution of $\bar{C}_{3}(x, p)$. After establishing the basic equations, we will first solver for $\bar{C}_{3}(x, p)$. In order to simplify the solutions, we denote

$$
\begin{aligned}
& h_{1}=l_{2}-l_{1}, \\
& h_{2}=l_{3}-l_{2}, \\
& \sigma_{1}=\sqrt{\frac{d_{1}}{d_{2}}},
\end{aligned}
$$

$$
\begin{aligned}
\sigma_{2} & =\sqrt{\frac{d_{2}}{d_{3}}}, \\
\tau_{1} & =\frac{D_{1}}{D_{2}}, \\
\tau_{2} & =\frac{D_{2}}{D_{3}} \\
X_{i} & \triangleq X_{i}(p)=\cosh q_{i}\left(l_{i+1}-l_{i}\right)=\cosh q_{i} h_{i}, \quad(i=1,2), \\
Y_{i} & \triangleq Y_{i}(p)=\sinh q_{i}\left(l_{i+1}-l_{i}\right)=\sinh q_{i} h_{i}, \quad(i=1,2),
\end{aligned}
$$




$$
\begin{aligned}
& A=\cosh q_{3}\left(x-l_{3}\right), \\
& B=\sinh q_{3}\left(x-l_{3}\right) .
\end{aligned}
$$

From (29), (35), and (36), we have

$$
\begin{aligned}
\left(\begin{array}{c}
\bar{C}_{3}(x, p)-\frac{C_{0}}{p} \\
\bar{J}_{3}(x, p)
\end{array}\right)= & \left(\begin{array}{cc}
A & -\frac{1}{D_{3} q_{3}} B \\
-D_{3} q_{3} B & A
\end{array}\right)\left(\begin{array}{cc}
X_{2} & -\frac{1}{D_{2} q_{2}} Y_{2} \\
-D_{2} q_{2} Y_{2} & X_{2}
\end{array}\right)\left(\begin{array}{cc}
X_{1} & -\frac{1}{D_{1} q_{1}} Y_{1} \\
-D_{1} q_{1} Y_{1} & X_{1}
\end{array}\right)\left(\begin{array}{c}
\bar{C}_{s}-\frac{C_{0}}{p} \\
\bar{J}_{0}
\end{array}\right) \\
= & \left(\bar{C}_{s}-\frac{C_{0}}{p}\right)\left(\begin{array}{c}
X_{1} X_{2} A+\frac{D_{1} q_{1}}{D_{2} q_{2}} Y_{1} Y_{2} A+\frac{D_{2} q_{2}}{D_{3} q_{3}} X_{1} Y_{2} B+\frac{D_{1} q_{1}}{D_{3} q_{3}} Y_{1} X_{2} B \\
-D_{3} q_{3} X_{1} X_{2} B-\frac{D_{1} q_{1} D_{3} q_{3}}{D_{2} q_{2}} Y_{1} Y_{2} B-D_{1} q_{1} Y_{1} X_{2} A-D_{2} q_{2} X_{1} Y_{2} A
\end{array}\right) \\
& +\bar{J}_{0}\left(\begin{array}{c}
-\frac{1}{D_{1} q_{1}} Y_{1} X_{2} A-\frac{1}{D_{2} q_{2}} X_{1} Y_{2} A-\frac{D_{2} q_{2}}{D_{1} q_{1} D_{3} q_{3}} Y_{1} Y_{2} B-\frac{1}{D_{3} q_{3}} X_{1} X_{2} B \\
\frac{D_{3} q_{3}}{D_{1} q_{1}} Y_{1} X_{2} B+\frac{D_{3} q_{3}}{D_{2} q_{2}} X_{1} Y_{2} B+\frac{D_{2} q_{2}}{D_{1} q_{1}} Y_{1} Y_{2} A+X_{1} X_{2} A
\end{array}\right) .
\end{aligned}
$$

Because of (32), the sum of the coefficients of $A$ and the coefficients of $B$ must equal zero in $\bar{C}_{3}(x, p)$. That is,

$$
\bar{J}_{0}=\frac{X_{1} X_{2}+\left(D_{1} q_{1} / D_{2} q_{2}\right) Y_{1} Y_{2}+\left(D_{2} q_{2} / D_{3} q_{3}\right) X_{1} Y_{2}+\left(D_{1} q_{1} / D_{3} q_{3}\right) Y_{1} X_{2}}{\left(1 / D_{1} q_{1}\right) Y_{1} X_{2}+\left(1 / D_{2} q_{2}\right) X_{1} Y_{2}+\left(D_{2} q_{2} / D_{1} q_{1} D_{3} q_{3}\right) Y_{1} Y_{2}+\left(1 / D_{3} q_{3}\right) X_{1} X_{2}}\left(\bar{C}_{s}-\frac{C_{0}}{p}\right) .
$$

Substituting (39) and (17) into (38), we have

$$
\begin{aligned}
\bar{C}_{3}(x, p) & =\frac{C_{0}}{p}+\left(\bar{C}_{s}-\frac{C_{0}}{p}\right) \frac{e^{-q_{3}\left(x-l_{3}\right)}}{Z} \\
& =\frac{C_{0}}{p}+\left(C_{s}-C_{0}\right) \frac{e^{-q_{3}\left(x-l_{3}\right)}}{p Z}
\end{aligned}
$$

where

$$
\begin{aligned}
Z= & \frac{\sigma_{1} \sigma_{2}}{\tau_{1} \tau_{2}} \sinh \left(\sqrt{\frac{p}{d_{1}}} h_{1}\right) \cosh \left(\sqrt{\frac{p}{d_{1}}} \sigma_{1} h_{2}\right) \\
& +\frac{\sigma_{2}}{\tau_{2}} \cosh \sqrt{\frac{p}{d_{1}}}\left(l_{2}-l_{1}\right) \sinh \left(\sqrt{\frac{p}{d_{1}}} \sigma_{1} h_{2}\right) \\
& +\frac{\sigma_{1}}{\tau_{1}} \sinh \left(\sqrt{\frac{p}{d_{1}}} h_{1}\right) \sinh \left(\sqrt{\frac{p}{d_{1}}} \sigma_{1} h_{2}\right) \\
& +\cosh \left(\sqrt{\frac{p}{d_{1}}} h_{1}\right) \cosh \left(\sqrt{\frac{p}{d_{1}}} \sigma_{1} h_{2}\right) .
\end{aligned}
$$

3.5. Inverse Laplace Transform. The complex inversion formula [7] is

$$
\begin{aligned}
C_{3}(x, t) & =\mathscr{L}^{-1}\left(\bar{C}_{3}(x, p)\right) \\
& =\frac{1}{2 \pi i} \int_{\gamma-i \infty}^{\gamma+i \infty} \bar{C}_{3}(x, p) e^{p t} d p .
\end{aligned}
$$

Substituting (40) into (42), we have

$$
C_{3}(x, t)=C_{0}+\left(C_{s}-C_{0}\right) \frac{1}{2 \pi i} \int_{\gamma-i \infty}^{\gamma+i \infty} \frac{e^{-q_{3}\left(x-l_{3}\right)}}{p Z} e^{p t} d p .
$$

It can be proved that the integrand in (43) only has a branch point at $p=0$. So we can use the contour in Figure 2. The line integral in (43) is found to be equal to the integral over the small circle about the origin together with the integrals over $C D$ and $E F$. That is,

$$
\begin{aligned}
& C_{3}(x, t)=C_{0} \\
& +\frac{\left(C_{s}-C_{0}\right)}{2 \pi i}\left(\lim _{\substack{R \rightarrow \infty \\
\varepsilon \rightarrow 0}}\left(\int_{E F}+\int_{C D}+\int_{D G E}\right) \frac{e^{-q_{3}\left(x-l_{3}\right)}}{p Z}\right. \\
& \left.\cdot e^{p t} d p\right) .
\end{aligned}
$$

According to the residue theorem [7], we have

$$
\begin{aligned}
& \frac{1}{2 \pi i \varepsilon \rightarrow 0} \lim _{D G E} \frac{e^{-q_{3}\left(x-l_{3}\right)}}{p Z} e^{p t} d p \\
& =\lim _{p \rightarrow 0}(p-0) \frac{e^{-q_{3}\left(x-l_{3}\right)}}{p Z} e^{p t}=1 .
\end{aligned}
$$

Denote $P_{1}(u)=\cos \left(h_{1} u\right), P_{2}(u)=\cos \left(\sigma_{1} h_{2} u\right), Q_{1}(u)=$ $\sin \left(h_{1} u\right)$, and $Q_{2}(u)=\sin \left(\sigma_{1} h_{2} u\right)$.

Putting $p=d_{1} u^{2} e^{i \pi}$ on $E F$ and $p=d_{1} u^{2} e^{-i \pi}$ on $C D$, the contribution from $C D$ and $E F$ becomes 


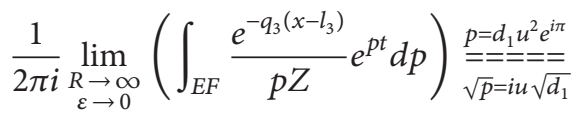

$$
\begin{aligned}
& =\frac{1}{\pi i} \int_{0}^{\infty} \frac{e^{-u^{2} d_{1} t} e^{-i u \sigma_{1} \sigma_{2}\left(x-l_{3}\right)}}{u\left(i\left(\sigma_{1} \sigma_{2} / \tau_{1} \tau_{2}\right) Q_{1}(u) P_{2}(u)+i\left(\sigma_{2} / \tau_{2}\right) P_{1}(u) Q_{2}(u)-\left(\sigma_{1} / \tau_{1}\right) P_{1}(u) P_{2}(u)+Q_{1}(u) Q_{2}(u)\right)} d u
\end{aligned}
$$

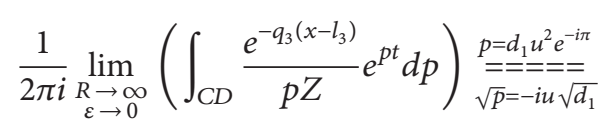

$$
\begin{aligned}
& =-\frac{1}{\pi i} \int_{0}^{\infty} \frac{e^{-u^{2} d_{1} t} e^{i u \sigma_{1} \sigma_{2}\left(x-l_{3}\right)}}{u\left(-i\left(\sigma_{1} \sigma_{2} / \tau_{1} \tau_{2}\right) Q_{1}(u) P_{2}(u)-i\left(\sigma_{2} / \tau_{2}\right) P_{1}(u) Q_{2}(u)-\left(\sigma_{1} / \tau_{1}\right) P_{1}(u) P_{2}(u)+Q_{1}(u) Q_{2}(u)\right)} d u \text {, }
\end{aligned}
$$

which are mutually conjugated. Consequently, denote

$$
\begin{aligned}
& W(u)=-\frac{\sigma_{1}}{\tau_{1}} P_{1}(u) P_{2}(u)+Q_{1}(u) Q_{2}(u), \\
& V(u)=\frac{\sigma_{1} \sigma_{2}}{\tau_{1} \tau_{2}} Q_{1}(u) P_{2}(u)+\frac{\sigma_{2}}{\tau_{2}} P_{1}(u) Q_{2}(u),
\end{aligned}
$$

$$
F(x, t, u)=\frac{e^{-u^{2} d_{1} t}\left[\cos \left(\sigma_{1} \sigma_{2} u\left(x-l_{3}\right)\right) V(u)+\sin \left(\sigma_{1} \sigma_{2} u\left(x-l_{3}\right)\right) W(u)\right]}{u\left(W^{2}(u)+V^{2}(u)\right)} .
$$

and we have

$$
C_{3}(x, t)=C_{s}-\frac{2\left(C_{s}-C_{0}\right)}{\pi} \int_{0}^{\infty} F(x, t, u) d u
$$

where
This is the analytical solution of (5)-(14), the chloride concentration profiles in the porous media with two layers of protection under constant boundary conditions.

\section{Nonconstant Boundary for \\ the Two-Protective-Layered Systems}

In reality, the boundary condition (the chloride concentration) on the surface of a bridge structure may not be constant. In this case, other boundary conditions should be considered. We can change constant boundary condition (13) to a nonconstant boundary condition, such as

$$
C_{1}\left(l_{1}, t\right)=g(t)=C_{s}+\int_{0}^{t} h(z) d z
$$

where $C_{s}$ is a constant and $h(t)(t \geq 0)$ is a continuous function; we can also obtain the analytical solution for the diffusion equation with boundary condition (50). Now, we consider (5)-(12), (50), and (14). Equation (17) becomes

$$
\bar{C}_{s}=\mathscr{L}(g(t))=\frac{\mathscr{L}(h(t))+C_{s}}{p},
$$

which is the Laplace transform of $C_{1}\left(l_{1}, t\right)$. The derivation is the same as in Section 3, which will not be repeated here. Equation (40) with the new boundary condition becomes

$$
\begin{aligned}
\bar{C}_{3}(x, p) & =\frac{C_{0}}{p}+\left(\bar{C}_{s}-\frac{C_{0}}{p}\right) \frac{e^{-q_{3}\left(x-l_{3}\right)}}{Z} \\
& =\frac{C_{0}}{p}+\left(\mathscr{L}(g(t))-C_{0}\right) \frac{e^{-q_{3}\left(x-l_{3}\right)}}{p Z} .
\end{aligned}
$$

By the inverse Laplace transform, we have

$$
\begin{aligned}
C_{3}(x, t)= & \mathscr{L}^{-1}\left(\bar{C}_{3}(x, p)\right) \\
= & C_{0}+\mathscr{L}^{-1}\left(\frac{\mathscr{L}(h(t)) e^{-\sqrt{p / d_{3}}\left(x-l_{3}\right)}}{p Z}\right) \\
& +\mathscr{L}^{-1}\left(\frac{\left(C_{s}-C_{0}\right) e^{-\sqrt{p / d_{3}}\left(x-l_{3}\right)}}{p Z}\right) .
\end{aligned}
$$

From Section 3.5, we know

$$
\mathscr{L}^{-1}\left(\frac{e^{-\sqrt{p / d_{3}}\left(x-l_{3}\right)}}{p Z}\right)=1-\frac{2}{\pi} \int_{0}^{\infty} F(x, t, u) d u .
$$




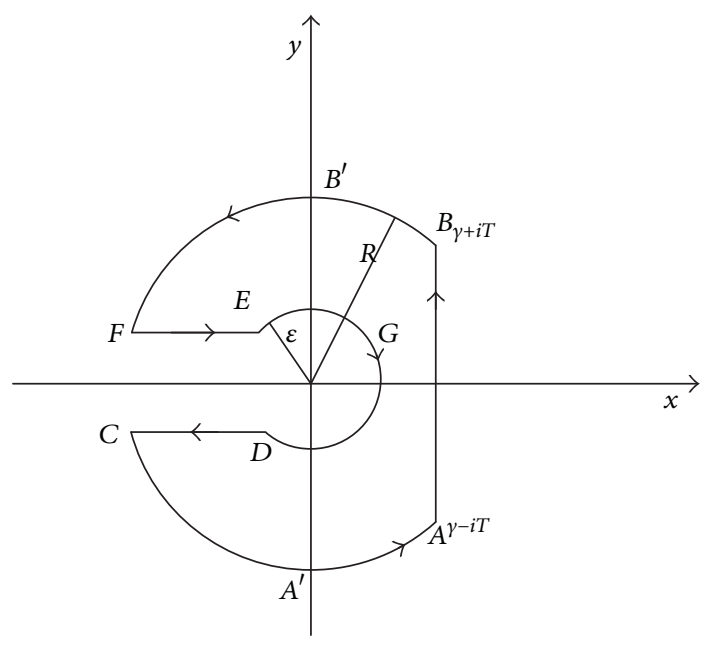

FIGURE 2: Integral contour.

Substituting (54) into (53) and by using the convolution theorem [7], we have

$$
\begin{aligned}
C_{3}(x, t) & \\
= & C_{0}+\mathscr{L}^{-1}\left(\mathscr{L}(h(t)) \frac{e^{-\sqrt{p / d_{3}}\left(x-l_{3}\right)}}{p Z}\right) \\
& +\left(C_{s}-C_{0}\right)\left(1-\frac{2}{\pi} \int_{0}^{\infty} F(x, t, u) d u\right) \\
= & C_{0} \\
& +\int_{0}^{t}\left[h(t-z)\left(1-\frac{2}{\pi} \int_{0}^{\infty} F(x, z, u) d u\right)\right] d z \\
& \quad-\frac{2\left(C_{s}-C_{0}\right)}{\pi} \int_{0}^{\infty} F(x, t, u) d u,
\end{aligned}
$$

where $F(x, t, u)$ is defined in (49). It can be seen that the analytical solution, (48), is a special case of (55); that is, $h(t) \equiv$ 0 .

As an example, a linear boundary condition can be considered, which is a commonly used nonconstant boundary condition; that is,

$$
C_{1}\left(l_{1}, t\right)=g(t)=a t+b
$$

where $a, b$ are two constants. From (55), we can easily obtain the analytical solution of (5)-(12), (55), and (14), which is

$$
\begin{aligned}
C_{3}(x, t)= & a t+b-\frac{2\left(b-C_{0}\right)}{\pi} \int_{0}^{\infty} F(x, t, u) d u \\
& +\frac{2 a}{\pi D_{1}} \int_{0}^{\infty}\left(\frac{1-e^{u^{2} d_{1} t}}{u^{2}}\right) F(x, t, u) d u
\end{aligned}
$$

where $F(x, t, u)$ is defined in (49).

\section{Analytical Solution with $(n-1)$ \\ Protective Layered System $(n>3)$}

In Sections 3 and 4 we obtained the analytical solutions for the chloride diffusion equation of two protective layers under constant and nonconstant boundary conditions, respectively. We can further generalize the results to the chloride diffusion equation with $(n-1)$ protective layers under nonconstant boundary conditions.

5.1. Diffusion Model for the Multiple Layered System. Assuming the system has $n-1$ protective layers (totally $n$ layers including the concrete), as shown in Figure 3, the material parameters of each protective layer and the concrete are $d_{1}, d_{2}, \ldots, d_{n}$ and $D_{1}, D_{2}, \ldots, D_{n}$, respectively, and all material parameters are constants. The corresponding concentrations of chloride ions are $C_{1}(x, t), C_{2}(x, t), \ldots, C_{n}(x, t)$, respectively. The chloride diffusion equations with $(n-1)$ protective layers are similar to those in Section 2:

$$
\begin{gathered}
\frac{\partial C_{1}}{\partial t}=d_{1} \frac{\partial^{2} C_{1}}{\partial x^{2}}, \quad l_{1} \leq x \leq l_{2} \\
C_{1}\left(l_{1}, t\right)=g(t)=C_{s}+\int_{0}^{t} h(t) d t \\
C_{1}(x, 0)=C_{0} \\
C_{1}(\infty, t)<\infty
\end{gathered}
$$

(The first layer)

$$
\begin{gathered}
\frac{\partial C_{2}}{\partial t}=d_{2} \frac{\partial^{2} C_{2}}{\partial x^{2}}, \quad l_{2} \leq x \leq l_{3}, \\
C_{2}(x, 0)=C_{0}, \\
C_{2}(\infty, t)<\infty, \\
C_{1}\left(l_{2}, t\right)=C_{2}\left(l_{2}, t\right), \\
\left.D_{1} \frac{\partial C_{1}}{\partial x}\right|_{x=l_{2}}=\left.D_{2} \frac{\partial C_{2}}{\partial x}\right|_{x=l_{2}},
\end{gathered}
$$

(The second layer)

$$
\begin{gathered}
\frac{\partial C_{n-1}}{\partial t}=d_{n-1} \frac{\partial^{2} C_{n-1}}{\partial x^{2}}, \quad l_{n-1} \leq x \leq l_{n}, \\
C_{n-1}(x, 0)=C_{0}, \\
C_{n-1}(\infty, t)<\infty \\
C_{n-2}\left(l_{n-1}, t\right)=C_{n-1}\left(l_{n-1}, t\right), \\
\left.D_{n-2} \frac{\partial C_{n-2}}{\partial x}\right|_{x=l_{n-1}}=\left.D_{n-1} \frac{\partial C_{n-1}}{\partial x}\right|_{x=l_{n-1}},
\end{gathered}
$$

(The $(n-1)$ th layer),

$$
\begin{gathered}
\frac{\partial C_{n}}{\partial t}=d_{n} \frac{\partial^{2} C_{n}}{\partial x^{2}}, \quad l_{n} \leq x, \\
C_{n-1}(x, 0)=C_{0}, \\
C_{n}(\infty, t)=C_{0}, \\
C_{n-1}\left(l_{n}, t\right)=C_{n}\left(l_{n}, t\right),
\end{gathered}
$$




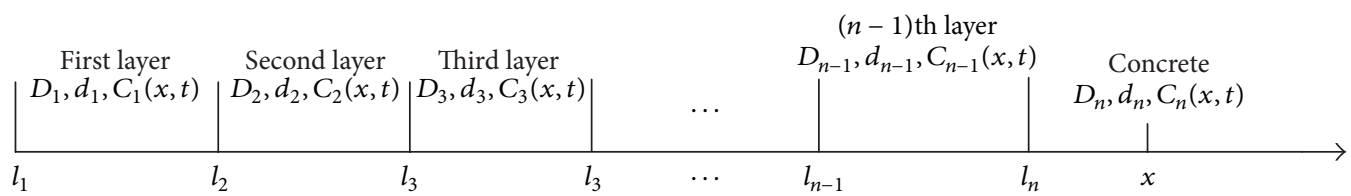

FIGURE 3: Concrete with $(n-1)$ layers of protection.

$$
\left.D_{n-1} \frac{\partial C_{n-1}}{\partial x}\right|_{x=l_{n}}=\left.D_{n} \frac{\partial C_{n}}{\partial x}\right|_{x=l_{n}}
$$

(Concrete).

5.2. Analytical Solution for the Multiple Layered System. We first introduce the denotations. Denote

$$
\begin{gathered}
h_{i}=l_{i+1}-l_{i}, \\
\sigma_{i}=\sqrt{\frac{d_{i}}{d_{i+1}}} \\
\tau_{i}=\frac{D_{i}}{D_{i+1}} \\
\alpha_{1}=1, \quad i=1,2, \ldots, n-1, \\
\alpha_{2}=\sigma_{1}, \\
\alpha_{3}=\sigma_{1} \sigma_{2} \\
\vdots \\
\alpha_{k}=\sigma_{1} \sigma_{2} \cdots \sigma_{k-1}, \\
\vdots \\
\alpha_{n-1}=\sigma_{1} \sigma_{2} \cdots \sigma_{n-2}, \\
\beta_{1}=1, \\
\beta_{2} \cdots \sigma_{n-1},
\end{gathered}
$$

$$
\beta_{3}=\tau_{1} \tau_{2}
$$$$
\beta_{k}=\tau_{1} \tau_{2} \cdots \tau_{k-1}
$$
$i=1,2, \ldots, n-1$.

Let

$$
\begin{gathered}
W_{n}(u)=P_{1} P_{2} \cdots P_{n-1}+\sum_{\substack{k \text { is even, } \\
n-1 \geq k>0}} S_{m_{1} m_{2} \cdots m_{k}}(-1)^{k / 2} \\
\cdot Q_{m_{1}} Q_{m_{2}} \cdots Q_{m_{k}} P_{m_{k+1}} P_{m_{k+2}} \cdots P_{m_{n-1}}, \\
V_{n}(u)=\sum_{\substack{k \text { is odd, } \\
n-1 \geq k>0}} R_{m_{1} m_{2} \cdots m_{k}}(-1)^{(k-1) / 2} \\
\cdot Q_{m_{1}} Q_{m_{2}} \cdots Q_{m_{k}} P_{m_{k+1}} P_{m_{k+2}} \cdots P_{m_{n-1}}
\end{gathered}
$$

where

(1) $m_{1}, m_{2}, \ldots, m_{n-1}$ is a permutation of $1,2, \ldots, n-1$,

(2) $m_{1}>m_{2}>\cdots>m_{k}$,

(3) $S_{m_{1} m_{2} \cdots m_{k}}=\frac{\alpha_{m_{k-1}} \beta_{m_{k}}}{\alpha_{m_{k}} \beta_{m_{k-1}}} \cdot \frac{\alpha_{m_{k-3}} \beta_{m_{k-2}}}{\alpha_{m_{k-2}} \beta_{m_{k-3}}} \cdots \frac{\alpha_{m_{1}} \beta_{m_{2}}}{\alpha_{m_{2}} \beta_{m_{1}}}, k$ is even,

(4) $R_{m_{1} m_{2} \cdots m_{k}}= \begin{cases}\frac{\alpha_{n}}{\beta_{n}} \cdot \frac{\beta_{m_{1}}}{\alpha_{m_{1}}}, & \text { if } k=1, \\ \frac{\alpha_{n}}{\beta_{n}} \cdot \frac{\alpha_{m_{k-1}} \beta_{m_{k}}}{\alpha_{m_{k}} \beta_{m_{k-1}}} \cdot \frac{\alpha_{m_{k-3}} \beta_{m_{k-2}}}{\alpha_{m_{k-2}} \beta_{m_{k-3}}} \cdots \frac{\beta_{m_{3}} \alpha_{m_{2}}}{\beta_{m_{2}} \alpha_{m_{3}}} \cdot \frac{\beta_{m_{1}}}{\alpha_{m_{1}}}, & \text { if } k \geq 3, k \text { is odd. }\end{cases}$ 
The lengthy derivation will not be shown here, and the results will be listed. The analytical solution of (58) for the multiple layered system is

$$
\begin{aligned}
& C_{n}(x, t) \\
& =C_{s} \\
& \quad+\int_{0}^{t}\left[h(t-z)\left(1-\frac{2}{\pi} \int_{0}^{\infty} F_{n}(x, z, u) d u\right)\right] d z \\
& \quad-\frac{2\left(C_{s}-C_{0}\right)}{\pi} \int_{0}^{\infty} F_{n}(x, t, u) d u,
\end{aligned}
$$

where

$$
\begin{aligned}
& F_{n}(x, t, u) \\
& =\frac{e^{-u^{2} d_{1} t}\left[\cos \left(\alpha_{n} u\left(x-l_{n}\right)\right) V_{n}(u)+\sin \left(\alpha_{n} u\left(x-l_{n}\right)\right) W_{n}(u)\right]}{u\left(W_{n}^{2}(u)+V_{n}^{2}(u)\right)} .
\end{aligned}
$$

\section{Heat Conduction in the Two-Protective-Layered System}

Heat conduction equation has the same form of equation as the chloride diffusion shown above, which is a parabolic partial differential equation. The difference lies in the different transport processes, which can be characterized by the different material parameters involved in the equations. Therefore, mathematically, we can expand our results to the heat conduction problem. As an example, we present the analytical solution of heat conduction equation in a system with two protective layers under nonconstant boundary conditions. Another reason for us to extend our results to the heat conduction is that an experiment can be relatively easier set up and conducted for the heat conduction than for the chloride diffusion (which is a very slow process). Using the test results of heat conduction, our analytical model can be verified.

6.1. Heat Conduction Model. For the heat conduction problem, the material parameters are heat capacity $c$, density $\rho$, and thermal conductivity $K$. The product of heat capacity and density, $c \rho$, is equivalent to the chloride binding capacity in the chloride diffusion equation, and the thermal conductivity $K$ is equivalent to the chloride diffusivity. A parameter similar to the chloride diffusion coefficient $d$ can be defined here for the heat conduction, which is $\kappa$ as shown in (64). $\kappa$ may be called heat diffusion coefficient. For the first layer, the second layer, and the concrete, we denote the material parameters as $\kappa_{i}, K_{i}, c_{i}$, and $\rho_{i}(i=1,2,3)$, respectively, and

$$
\kappa_{i}=\frac{K_{i}}{\rho_{i} c_{i}}, \quad i=1,2,3 .
$$

The temperatures of the first layer, the second layer, and the concrete are $T_{1}(x, t), T_{2}(x, t)$, and $T_{3}(x, t)$, which are governed by the following equations:

$$
\begin{array}{ll}
\frac{\partial T_{1}}{\partial t}=\kappa_{1} \frac{\partial^{2} T_{1}}{\partial x^{2}}, & l_{1} \leq x \leq l_{2}, \\
\frac{\partial T_{2}}{\partial t}=\kappa_{2} \frac{\partial^{2} T_{2}}{\partial x^{2}}, & l_{2} \leq x \leq l_{3}, \\
\frac{\partial T_{3}}{\partial t}=\kappa_{3} \frac{\partial^{2} T_{3}}{\partial x^{2}}, & x \geq l_{3} .
\end{array}
$$

At the two interfaces, they satisfy the continuity conditions:

$$
\begin{aligned}
T_{1}\left(l_{2}, t\right) & =T_{2}\left(l_{2}, t\right), \\
\left.K_{1} \frac{\partial T_{1}}{\partial x}\right|_{x=l_{2}} & =\left.K_{2} \frac{\partial T_{2}}{\partial x}\right|_{x=l_{2}}, \\
T_{2}\left(l_{3}, t\right) & =T_{3}\left(l_{3}, t\right), \\
\left.K_{2} \frac{\partial T_{2}}{\partial x}\right|_{x=l_{3}} & =\left.K_{3} \frac{\partial T_{3}}{\partial x}\right|_{x=l_{3}} .
\end{aligned}
$$

The initial conditions are

$$
T_{1}(x, 0)=T_{2}(x, 0)=T_{3}(x, 0)=T_{0} .
$$

The nonconstant boundary conditions are

$$
\begin{aligned}
& T_{1}\left(l_{1}, t\right)=g(t)=T_{s}+\int_{0}^{t} h(z) d z, \\
& T_{1}(\infty, t)<\infty, \\
& T_{2}(\infty, t)<\infty, \\
& T_{3}(\infty, t)=T_{0} .
\end{aligned}
$$

$T_{0}$ and $T_{s}$ are constants. These are the governing equations, initial and boundary conditions for the heat conduction problem of a two-protective-layered system.

6.2. Analytical Solution. Similar to the derivation in Section 3, we can obtain an analytical solution for (65)-(69) as follows. Denote $h_{1}=l_{2}-l_{1}, h_{2}=l_{3}-l_{2}, \widetilde{\sigma}_{1}=\sqrt{\kappa_{1} / \kappa_{2}}$, $\widetilde{\sigma}_{2}=\sqrt{\kappa_{2} / \kappa_{3}}, \widetilde{\tau}_{1}=K_{1} / K_{2}$, and $\widetilde{\tau}_{2}=K_{2} / K_{3}$, and further denote

$$
\begin{aligned}
\widetilde{P}_{1}(u) & =\cos \left(h_{1} u\right), \\
\widetilde{P}_{2}(u) & =\cos \left(\widetilde{\sigma}_{1} h_{2} u\right), \\
\widetilde{Q}_{1}(u) & =\sin \left(h_{1} u\right),
\end{aligned}
$$




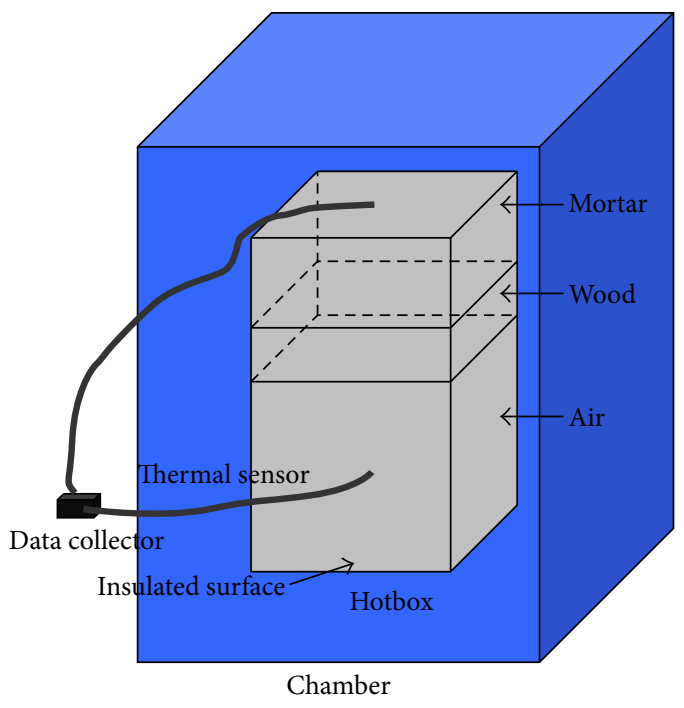

(a)

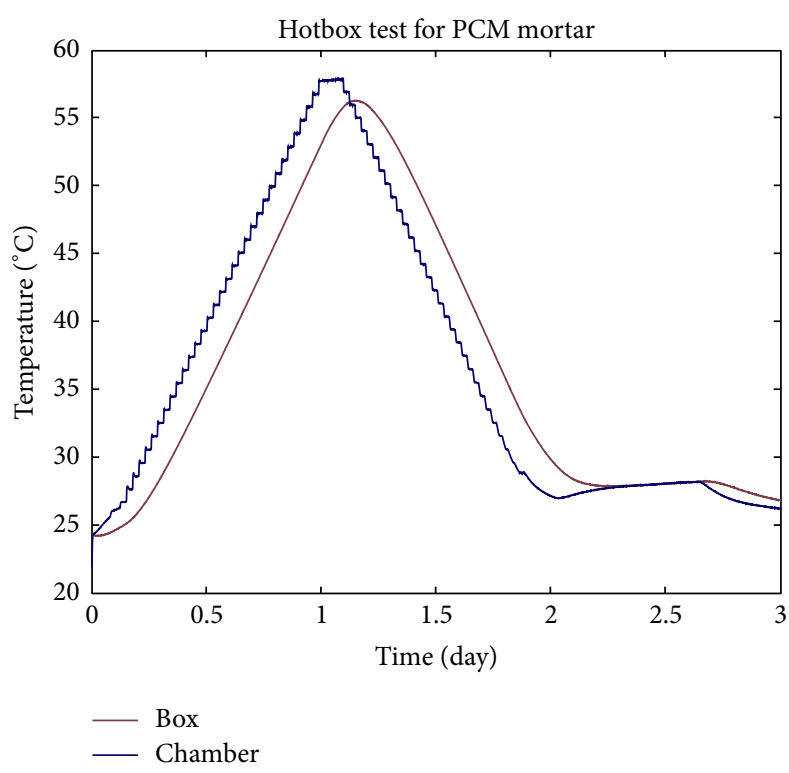

(b)

FIgURE 4: A heat conduction test: (a) experimental setup; (b) test results [8].

$\widetilde{Q}_{2}(u)=\sin \left(\widetilde{\sigma}_{1} h_{2} u\right)$

$$
\begin{aligned}
& \widetilde{W}(u)=-\frac{\widetilde{\sigma}_{1}}{\widetilde{\tau}_{1}} \widetilde{P}_{1}(u) \widetilde{P}_{2}(u)+\widetilde{Q}_{1}(u) \widetilde{Q}_{2}(u), \\
& \widetilde{V}(u)=\frac{\widetilde{\sigma}_{1} \widetilde{\sigma}_{2}}{\widetilde{\tau}_{1} \widetilde{\tau}_{2}} \widetilde{Q}_{1}(u) \widetilde{P}_{2}(u)+\frac{\widetilde{\sigma}_{2}}{\widetilde{\tau}_{2}} \widetilde{P}_{1}(u) \widetilde{Q}_{2}(u),
\end{aligned}
$$

in which the equal signs are from (64). We obtain

$$
\begin{aligned}
& T_{3}(x, t) \\
& =T_{s} \\
& \quad+\int_{0}^{t}\left[h(t-z)\left(1-\frac{2}{\pi} \int_{0}^{\infty} \widetilde{F}(x, z, u) d u\right)\right] d z \\
& \quad-\frac{2\left(T_{s}-T_{0}\right)}{\pi} \int_{0}^{\infty} \tilde{F}(x, t, u) d u,
\end{aligned}
$$

where

$$
\widetilde{F}(x, t, u)=\frac{e^{-u^{2} \kappa_{1} t}\left[\cos \left(\widetilde{\sigma}_{1} \widetilde{\sigma}_{2} u\left(x-l_{3}\right)\right) \widetilde{V}(u)+\sin \left(\widetilde{\sigma}_{1} \widetilde{\sigma}_{2} u\left(x-l_{3}\right)\right) \widetilde{W}(u)\right]}{u\left(\widetilde{W}^{2}(u)+\widetilde{V}^{2}(u)\right)},
$$

which is the analytical solution of the heat conduction model with a two-protective-layered system. We can easily generalize the results to the heat conduction problem of an $(n-1)$ layered system under nonconstant boundary conditions, which will not be listed here.

6.3. Numerical Example and Verification of the Solutions. A heat conduction test was conducted using a timber box (to simulate a residential house) [8]. An insulation material called premixed rubberized insulation mortar (PRIM) was applied on the exterior wall of the box. So, the wall has two layers: the timber board and the PRIM insulation layer. The box was placed in the environmental chamber, as shown in Figure 4(a). The chamber temperature was programmed to apply linear heating and linear cooling. Both chamber temperature and the temperature in the box were monitored, and the two temperature profiles were compared to examine the effectiveness of the insulation material. One of the results is shown in Figure 4(b). We simplified this problem to be a one-dimensional heat conduction problem through the twolayered wall of the timber box. The first layer is PRIM, with a thickness of one inch, and the second layer is wood, with a thickness of $11 / 16$ inches.

The average values of material properties for the PRIM, wood, and air are taken from literature as $\kappa_{1}=0.373 \mathrm{~mm}^{2} / \mathrm{s}$, $\kappa_{2}=0.139 \mathrm{~mm}^{2} / \mathrm{s}$, and $\kappa_{3}=22.000 \mathrm{~mm}^{2} / \mathrm{s}$, respectively. At the exterior boundary, $x=0 \mathrm{~mm}$; and at the two interfaces, we have $h_{1}=25.4 \mathrm{~mm}$ and $h_{2}=17.4625 \mathrm{~mm}$. For the linear 


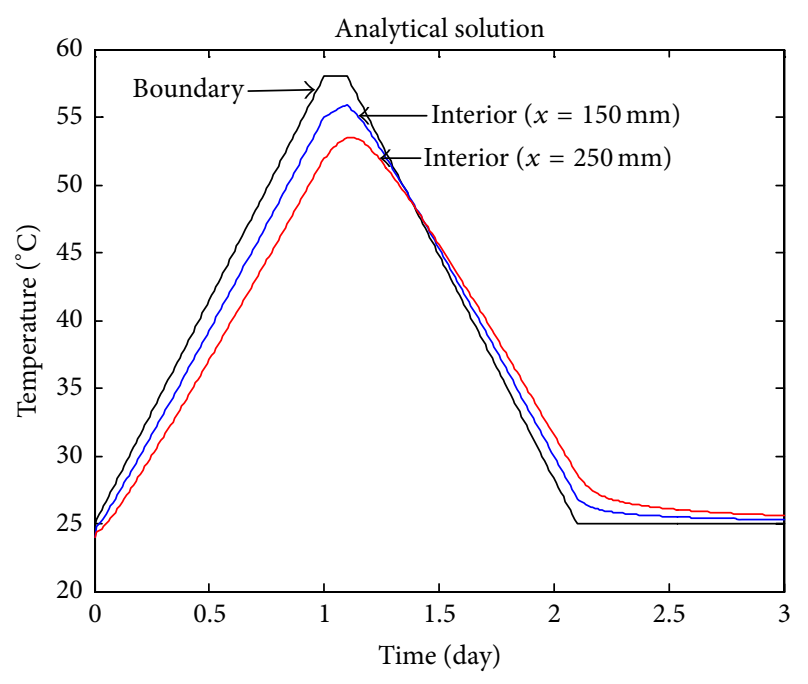

FIGURE 5: The interior temperatures calculated by the analytical solution (at $x=150 \mathrm{~mm}$ and $x=250 \mathrm{~mm}$ ).

boundary conditions, we approximated the temperatures in the chamber (outside of the box) by the following functions:

$$
g(t)= \begin{cases}33 t+25, & 0 \leq t<1 \\ 58, & 1 \leq t<1.1 \\ -33 t+94.3, & 1.1 \leq t<2.1 \\ 25, & 2.1 \leq t<3\end{cases}
$$

which are the boundary conditions as shown in (68), with $T_{s}=25^{\circ} \mathrm{C}$. And $T_{0}=24^{\circ} \mathrm{C}$.

Substituting the above parameters $k_{i}(i=1,2,3), h_{i}(i=$ $1,2), T_{0}, T_{s}, g(t)$ to (71) and by using the Gaussian quadrature integration scheme, the interior temperatures at $x=150 \mathrm{~mm}$ and $x=250 \mathrm{~mm}$ were calculated and shown in Figure 5 . Comparing the measured curves in Figure 4 with the calculated curves in Figure 5, one can see that the analytical solutions and the experimental results share the same trends, which means that the solution, (71), can predict the heat conduction problem quite well. Since the exact values of the thermal properties of the materials used in the test were not available, we can only compare the basic trend of the model predictions with the test data.

\section{Conclusions}

In this paper, the chloride ion diffusion equations were established for concrete structures with multiple layers of protective system. By using Laplace transformation, we first obtained an analytical solution for chloride concentration profiles in two-layered system and then extended the solution to multiple layered systems with nonconstant boundary conditions, including the constant boundary and linear boundary conditions.

Because ionic diffusion in saturated media and heat conduction are governed by the same form of partial differential equations with different materials parameters, we further extended our results to the analytical solution of heat conduction equation with multiple layered systems under nonconstant boundary conditions. We used the heat condition solution of a two-layered system as a numerical example. The numerical results were compared with available test data. The basic trends of the analytical solution and the test data agreed quite well.

The analytical solutions developed in this paper are very useful in the practice for predicting chloride penetration into concrete structures with multiple layers of protective systems. This method of analysis will be a great tool to estimate the time for the chloride ions to reach the critical chloride concentration at the rebar level and thus an effective remedy measure can be taken to avoid the corrosion damage in reinforced concrete structures. The analytical solution can also be used in general for any ionic diffusion problems in multilayered systems of porous media. As shown in the paper, the solution can be extended to deal with the heat condition in multilayered porous media.

\section{Conflict of Interests}

The authors declare that there is no conflict of interests regarding the publication of this paper.

\section{Acknowledgments}

This work was supported by Academic Human Resources Development in Institutions of Higher Learning under the Jurisdiction of Beijing Municipality (PHR201107123) and 2011 Science and Technology Research Project of Beijing Municipal Education Commission (KM201110016012). This work was also supported by Colorado Department of Transportation (Contract no. 12 HAA 38520, SAP no. 271001603).

\section{References}

[1] E. L. Cussler, Diffusion, Mass Transfer in Fluid Systems, Cambridge University Press, Cambridge, UK, 1997.

[2] M. Collepardi, A. Marcialis, and R. Turrizzani, "Penetration of chloride ions into cement pastes and concretes," Journal of the American Ceramic Society, vol. 55, no. 10, pp. 534-535, 1972.

[3] J. S. Kong, A. N. Ababneh, D. M. Frangopol, and Y. Xi, "Reliability analysis of chloride penetration in saturated concrete," Probabilistic Engineering Mechanics, vol. 17, no. 3, pp. 305-315, 2002.

[4] Y. Xi and Z. P. Bažant, "Modeling chloride penetration in saturated concrete," Journal of Materials in Civil Engineering, vol. 11, no. 1, pp. 58-65, 1999.

[5] N. Damrongwiriyanupap, L. Y. Li, and Y. Xi, "Coupled diffusion of multi-component chemicals in non-saturated concrete," Computers and Concrete, vol. 11, no. 3, pp. 201-222, 2013.

[6] H. S. Carslaw and J. C. Jaeger, Conduction of Heat in Solids, Oxford Science Publications, The Clarendon Press, New York, NY, USA, 2nd edition, 1988.

[7] R. S. Murray, Theory and Problems of Laplace Transforms, Schaum Publishing Company, 1965.

[8] Y. Xi, P. Newell, O. Na, and Y. Li, "Premixed rubberized insulation mortar (PRIM)," Tech. Rep. SESM 10-02, Department of Civil, Environmental and Architectural Engineering, University of Colorado Boulder, Boulder, Colo, USA, 2010. 


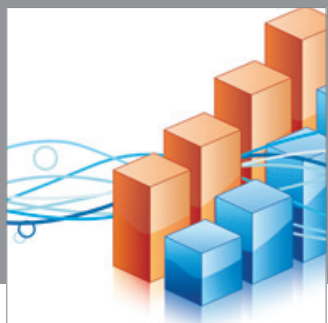

Advances in

Operations Research

mansans

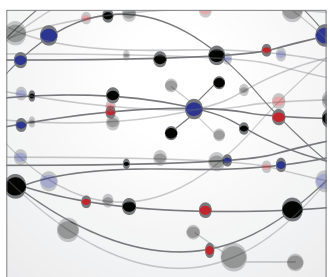

The Scientific World Journal
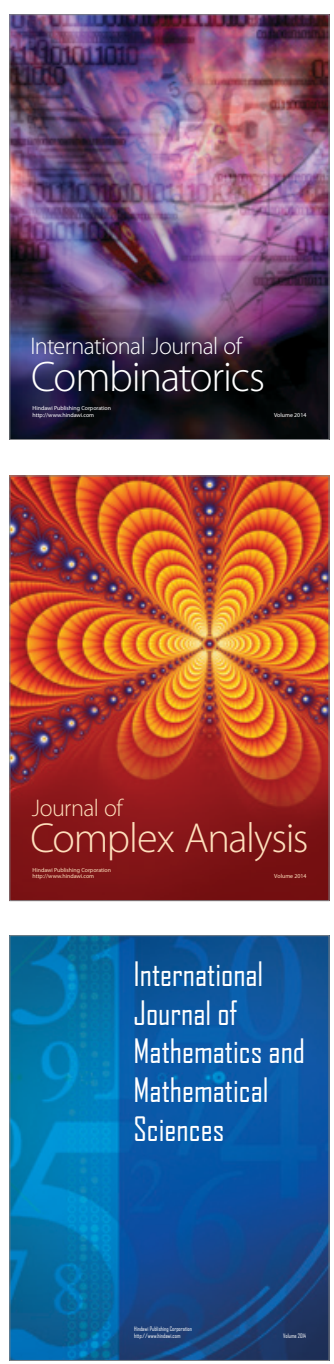
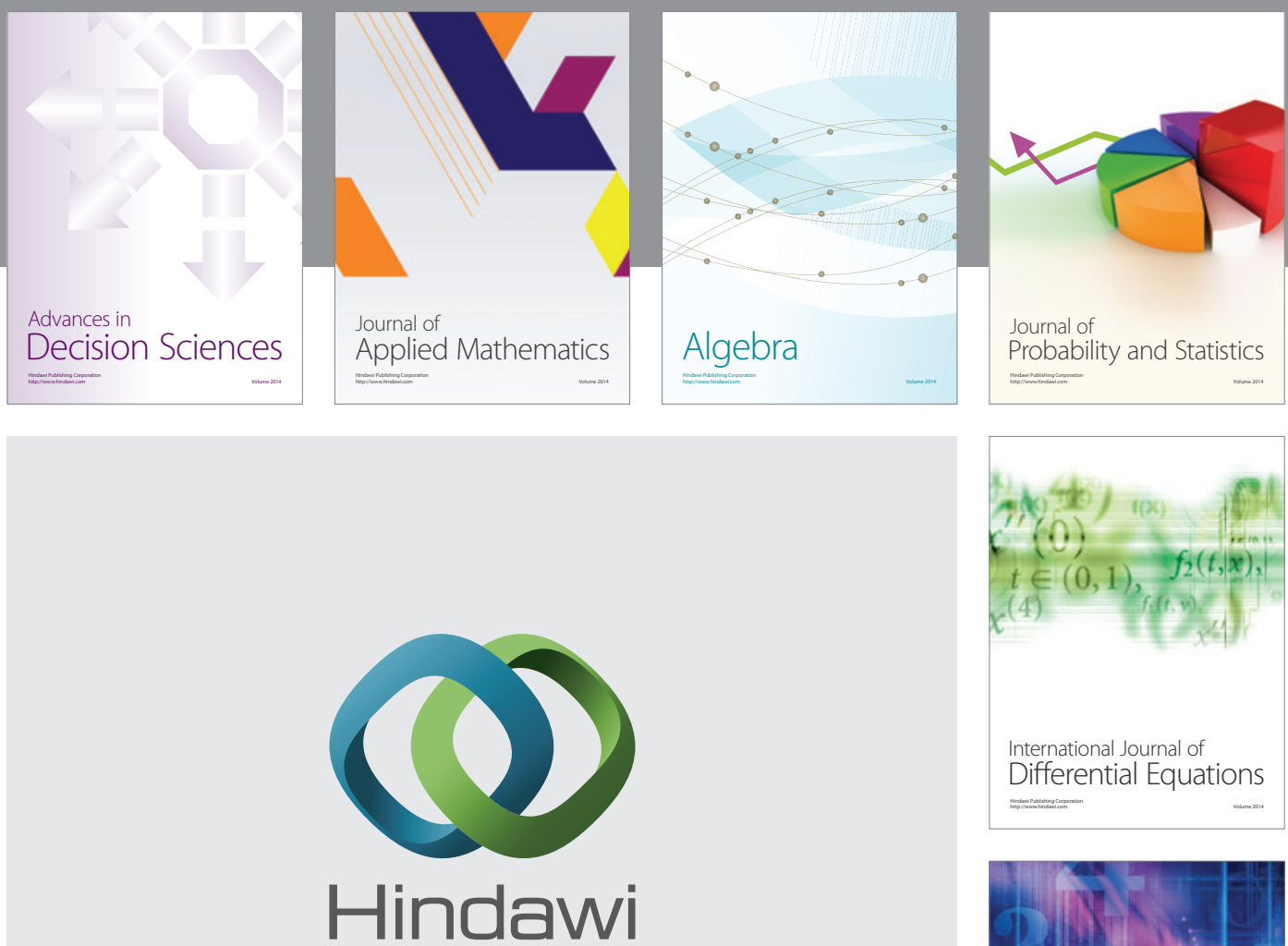

Submit your manuscripts at http://www.hindawi.com
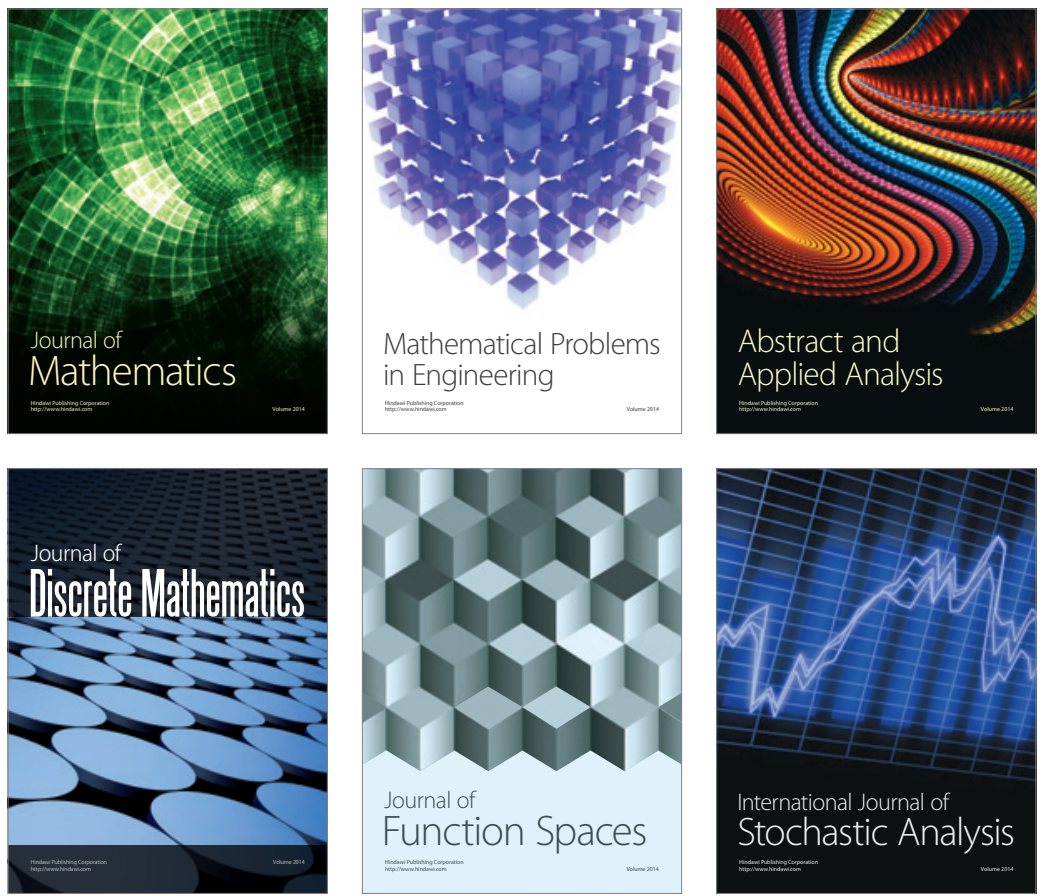

Journal of

Function Spaces

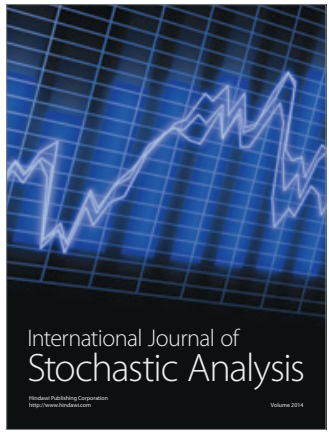

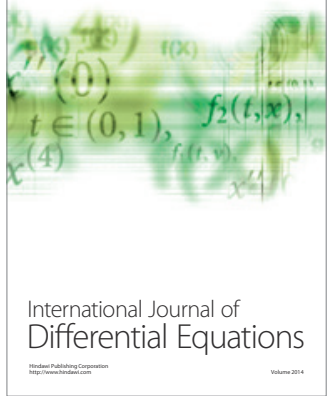
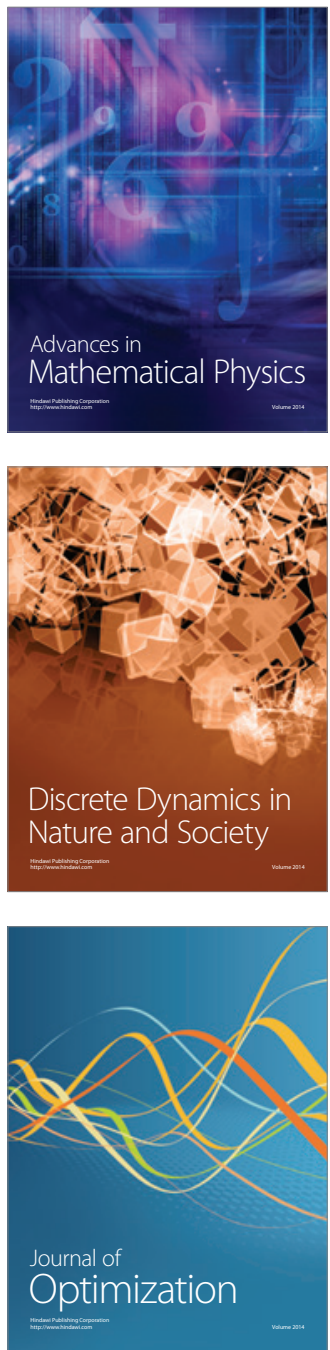